FERMILAB-PUB-94/160-T

$\sin 3426$

\title{
Large-Order Behavior and Nonperturbative Effects
}

\author{
Taekoon Lee \\ Fermi National Accelerator Laboratory \\ P.O. Box 500, Batavia, IL 60510
}

\begin{abstract}
We consider the energy-dependence of the large-order behavior of forward scattering amplitudes in theories having explicit mass parameters and instanton solutions. We show that in weak coupling theories the exponential part of the Espinosa-Ringwald type cross section can be determined by taking the minimum element of the perturbative series, suggesting that the series is asymptotic. This observation enables us to calculate the nonperturbative cross section by the perturbation of the Borel transform $\tilde{\sigma}(b)$ about the instanton-anti-instanton singularity. The anomalous cross section in the standard model using the leading energy-dependent $\tilde{\sigma}(b)$ is presented.
\end{abstract}

CERN LIBRARIES, GENEVA

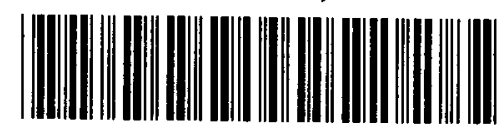

P00024033 


\section{Introduction}

Although there are extensive works for the large-order behavior of perturbation theory, its dependence on the external momenta is relatively unexplored. The only well known example on the momentum-dependence of the large-order behavior appears to be that of the scalar $\phi^{\mathbf{4}}$ theory. In the scalar theory, the large-order behavior of the r-point Green's function $G^{(r)}\left(p_{1}, \cdots, p_{r}\right)$ takes in general the form [1]

$$
\left\{G^{(r)}\left(p_{1}, \cdots, p_{r}\right)\right\}_{n} \sim C_{r}\left(p_{1}, \cdots, p_{r}\right) n ! n^{\nu-1} R^{n} \quad \text { for } \quad n \rightarrow \infty
$$

where $\nu$ and $R$ are constants and $C_{r}$ is independent of $n$. The momentum-dependence of the large-order behavior is factored out. Thus it is trivial. In section 2 we present examples of large-order behavior with interesting nontrivial momentum-dependence in theories having instanton solutions. Specifically, we calculate the large-order behavior of the forward scattering amplitudes induced by the instanton-anti-instanton singularity of the Borel transform in several models, including the standard model of electroweak interactions.

Aside from its intrinsic theoretical interest, the study of the energy-dependence of the large-order behavior is also interesting because it may find some application in giving a bound to the Espinosa-Ringwald type nonperturbative effects in high-energy scatterings. Generally, the perturbative expansion in the coupling $g$ of Green's functions in field theories is believed to be asymptotic. If it is indeed so, the nonperturbative effects associated with a Green's function may be estimated by taking the minimum element of the perturbative series. A well-known example is the quantum mechanical double-well potential problem. The 
minimum element of the perturbative series for the ground state energy is of order $e^{-2 S_{0}}[1]$, where $S_{0}$ is the instanton action, giving the correct exponential part of the instanton-antiinstanton contributions. Of course, the leading nonperturbative correction to the ground state energy comes not from the instanton-anti-instanton pairs but from the instantons, whose contributions the perturbative series does not account for. The reason for this is apparently related to the topology of the fluctuations. By definition, the perturbation in the coupling involves only fluctuations that begin and end at one of the classical vacua at time $t=\mp \infty$ respectively, to which the instantons do not belong. Thus the leading nonperturbatve corrections arising from the perurbative series are naturally expected to be those of the instanton-anti-instantons. This example suggests that at least for vacuum quantities that do not involve external parameters such as momenta, the nonperturbative effects associated with the fluctuations compatible with the perturbation in the coupling can be estimated by the minimum element of the perturbative series.

An extension of this idea to Green`s functions with large external momenta to estimate their nonperturbative content appears to be possible, at least in principle, for the theories that we consider in the following. We consider theories having explicit dimensional parameters and instanton solutions (including constrained instantons), and exclude QCD type interactions that have an infrared divergence associated with the instanton size. When the renormalon effects are neglected, the coefficients of the perturbative series of a Green's function can be determined by its Borel transform calculated in the instanton-anti-instanton background. Since the renormalons are associated with the Feynman diagrams having few in- 
termediate particles, they can be safely neglected for the instanton-induced nonperturbative effects that involve multiparticle production [2]. Therefore in this article the renormalons are not considered.

For several models, including the standard model, the Borel transform $\tilde{\sigma}(b)$ for the forward scattering amplitudes was calculated in [3] within the saddle point approximation. There, it was shown that the Borel transform has the instanton-anti-instanton singularity at $b=1$, as for the vacuum tunneling amplitudes, while the strength of the singularity generally depends on the c.m. energy. As is well known, the large-order behavior of perturbation theory is controlled by the functional behavior of the Borel transform near the singularity, and the perturbation of $\tilde{\sigma}(b)$ about the singularity provides a systematic approximation to calculate the perturbative asymptotics at large orders. We show in section 3 that the perturbative expression in $E / E_{0}$, where $E_{0}$ is the sphaleron energy, of the exponential part of the Espinosa-Ringwald type cross section [4] can be obtained by taking the minimum element of the perturbative series calculated by the expansion of $\tilde{\sigma}(b)$ about the Borel singularity. This suggests that the perturbative series in the coupling is indeed asymptotic.

Although the perturbation of $\tilde{\sigma}(b)$ about the Borel singularity gives identical perturbative expression for the Espinosa-Ringwald type cross section as in the KRT formalism [9], an important difference appears at high energies. In the KRT formalism, the Green's functions are evaluated in the instanton background to calculate the nonperturbative cross section, and the c.m. energy turns out to be the expansion parameter of the cross section. Since in the Borel transform method the energy is not the expansion parameter, it could possibly give 
a better description of the nonperturbative effects at high energies. Indeed, the anomalous cross section in the standard model calculated using the leading energy-dependent $\tilde{\sigma}(b)$ is exponentially suppressed, and thus unitarity is not violated, at all reasonably high energies. This, however, should be regarded as an interesting observation rather than a concrete conclusion, because, as we will see, the validity of the leading approximation breaks down at relatively low energies. Whether this interesting result, or at least the improved high-energy behavior of the anomalous cross section. will be preserved even when higher-order corrections are included is under investigation.

\section{Large-order behavior}

We calculate in this section the leading large-order behavior of the forward scattering amplitudes using the Borel transform $\tilde{\sigma}(b)$. The nontrivial energy-dependence of the large-order behavior is emphasized. Let us consider a theory having a dimensionless coupling $g$ and mass $m$. An extension to theories having more than one mass parameters should be straightforward. The Lagrangian is

$$
\mathcal{L}=\frac{1}{g} L(\phi, m)
$$

with $\phi$ representing generic fields. We assume that $m$ is independent of $g$, and $g$ can be always factored out of $L$ so that $L$ is independent of $g$. We also assume that there is an instanton solution with the action

$$
S_{I}=\frac{S_{0}}{g}
$$


where $S_{0}$ is a constant. Let us consider the perturbative expansion in the coupling $g$ of the two-body forward scattering amplitudes

$$
A\left(-p_{1},-p_{2}: p_{1}, p_{2}, g\right)=\sum_{n=0}^{\infty} a_{n} g^{n}
$$

As shown by Crutchfield II [5], the coefficients of the perturbative series can be determined by the Borel transform, $\tilde{\sigma}(b)$, defined by

$$
\bar{\sigma}(b)=\frac{1}{2 \pi} \int_{a-i \infty}^{a+i \infty} e^{z b} \sigma(g) d z
$$

where $z=2 S_{0} / g$, and $\sigma(g)$ is the imaginary part of the forward scattering amplitude calculated in the instanton-anti-instanton background. The Borel transform (5) is defined such that the instanton-anti-instanton singularity of the vacuum tunneling amplitudes is located at $b=1$. Note that $\sigma(g)$ is nothing but the Espinosa-Ringwald type cross section that has been extensivey studied in relation to the anomalous baryon number violation in the standard model $[6]$. Expanding $\tilde{\sigma}(b)$ at the origin. $b=0$.

$$
\tilde{\sigma}(b)=\sum_{n=0}^{\infty} c_{n} b^{n}
$$

we can obtain the coefficients $a_{n}$ by

$$
a_{n}=(n-1) ! c_{n-1}\left(\frac{1}{2 S_{0}}\right)^{n}
$$

Generally the Espinosa-Ringwald type cross section is given in the form

$$
\sigma(g) \sim\left(\frac{1}{g}\right)^{\nu} \exp \left(-\frac{2 S_{0}}{g} \mathcal{F}(x)\right) \sim z^{\nu} \exp (-z \mathcal{F}(x))
$$


and

$$
\mathcal{F}(x)=1-U(x)
$$

with $\nu$ a model-dependent constant, $x=\epsilon / z$, and $\epsilon=E / m$ where $E$ is the c.m. energy [6]. $U(x)$ can be calculated perturbatively in $x$ by doing perturbation about the instanton background, and its first few terms are known in several models [6. 8]. Let us now consider the large-order behavior of the standard model. In the standard model, $S_{0}=8 \pi^{2}, g=g_{w}^{2}$ and $m=m_{w}$ where $g_{w}, m_{w}$ are the weak-gauge boson coupling and the W-boson mass respectively. From [7]

$$
\nu=\frac{5}{6}
$$

For simplicity, we assume that the Weinberg angle vanishes so that electromagnetism decouples, and the Higgs coupling constant $\lambda$ is proportional to $g^{2}$. Then $U(x)$ is given by $[9]$

$$
U(x)=\frac{1}{2}(3 x)^{\frac{4}{3}}-\frac{3}{2} x^{2}+\cdots
$$

Substituting ( $(8)$ into $(5)$, we have

$$
\tilde{\sigma}(b) \sim \frac{1}{2 \pi} \int_{a-i \infty}^{a+i \infty} \exp [z(b-1+U(x))+\nu \ln z] d z
$$

which may be evaluated by the saddle point approximation. The equation for the saddle point $\bar{z}$, or equivalently $\bar{x}=t / \bar{z}$ is

$$
\begin{aligned}
1-b & =\frac{\nu}{\epsilon} \bar{x}+\left(1-\bar{x} \frac{\partial}{\partial \bar{x}}\right) U(\bar{x}) \\
& =\frac{\nu}{\epsilon} \bar{x}-\frac{1}{6}(3 \bar{x})^{\frac{4}{3}}+\frac{3}{2} \bar{x}^{2}+\cdots
\end{aligned}
$$


and the Borel transform is given by

$$
\tilde{\sigma}(b) \sim \frac{1}{\bar{x} \sqrt{1-\frac{\epsilon}{\nu} \bar{x} U^{\prime \prime}(\bar{x})}} \exp [\bar{z}(b-1+U(\bar{x}))+\nu \ln \bar{z}] .
$$

The Borel singularity occurs at $\bar{x}=0$, or equivalently at $b=1$ from (13), and solving (13) in series of $(1-b)$ we have

$$
\bar{x}(b)=\frac{\epsilon}{\nu}(1-b)\left(1+\frac{1}{6}\left(\frac{3 \epsilon}{\nu}\right)^{\frac{4}{3}}(1-b)^{\frac{1}{3}}+\cdots\right) .
$$

Thus the leading singular behavior of $\tilde{\sigma}(b)$ is given by

$$
\tilde{\sigma}(b) \sim(1-b)^{-(\nu+1)}
$$

which shows that the strength of the leading singularity is independent of the energy. Using (16) we find that at large orders the perturbative coefficients $a_{n}$ are given by

$$
a_{n} \rightarrow C(E) n ! n^{\nu-1}\left(\frac{1}{2 S_{0}}\right)^{n} \quad \text { for } \quad n \rightarrow \infty
$$

where $C(E)$ is a function of the energy. As in the scalar $\phi^{4}$ theory the energy-dependence of the leading perturbative asymptotics appears as a prefactor, and is thus trivial.

Nontrivial energy-dependence of the large-order behavior emerges in the case of the quantum mechanical double-well potential, the two-dimensional abelian Higgs model and the nonabelian Higgs model in three dimensions. For the double-well potential and the two-dimensional abelian Higgs model, the leading Borel transform $\tilde{\sigma}(b)$ from the similar calculation as in the standard model is given by [3]

$$
\tilde{\sigma}(b) \sim(1-b)^{-(\nu+1+e / l)}\left(\ln \frac{1}{(1-b)}\right)^{-\epsilon k / l} \quad \text { for } \quad b \rightarrow 1
$$


with $l=2, k=0$ for the double-well potential. and $l=1, k=1 / 2$ for the two-dimensional abelian Higgs model. For the nonabelian Higgs model in three dimensions,

$$
\tilde{\sigma}(b) \sim(1-b)^{-(2 \nu+3 / 2)} \exp \frac{\epsilon c^{2}}{1-b}
$$

where $c$ is the 't Hooft-Polyakov magnetic monolpole charge. Note the strong energydependence of the strength of the singularities in (18) and (19). The coefficients $c_{n}$ can be determined by

$$
\begin{aligned}
c_{n-1} & =\frac{1}{2 \pi i} \int_{C} \frac{\tilde{\sigma}(b)}{b^{n}} d b \\
& =\frac{1}{2 \pi i} \int_{C} \tilde{\sigma}(b) e^{-n \ln b} d b
\end{aligned}
$$

where $C$ is a path encircling the origin in the complex b-plane. With $\tilde{\sigma}(b)$ given in (18), equation (20) may be evaluated by the saddle point method for large $n$. It is easy to check that the saddle point for the double-well potential and the two-dimensional abelian Higgs model is given by

$$
\bar{b}=\frac{n}{n+\nu+1+\epsilon / l}\left(1+k \cdot O\left(\frac{1}{n \ln n}\right)\right)
$$

and the perturbative coefficients have the asymptotic form

$$
a_{n} \rightarrow C^{\prime}(E) n ! n^{(\nu-1+\epsilon / l)}(\ln n)^{-\epsilon k / l}\left(\frac{1}{2 S_{0}}\right)^{n} \quad \text { for } \quad n \rightarrow \infty
$$

where $C^{\prime}(E)$ is a function of the energy. A similar calculation for the nonabelian Higgs model in three dimensions gives.

$$
\bar{b}=1-c \sqrt{\frac{\epsilon}{n}}\left(1+O\left(\frac{1}{\sqrt{\epsilon n}}\right)\right)
$$


and

$$
a_{n} \rightarrow C^{\prime \prime}(E) n ! n^{(\nu-1)} e^{2 c \sqrt{\epsilon n}}\left(\frac{1}{2 S_{0}}\right)^{n} \quad \text { for } \quad n \rightarrow \infty
$$

where $C^{\prime \prime}(E)$ is a function of the energy. Note that

$$
\bar{b} \rightarrow 1 \text { for } n \rightarrow \infty
$$

in (21) and (23), which implies that the main contribution to the integral in (20) comes from the region near the singularity, where the approximate Borel transform in (18) and (19) are valid. Unlike the case of the scalar $\phi^{4}$ theory or the standard model, the perturbative asymptotics in (22) and (24) have a nontrivial energy-dependence. As shown in [3], the nature and strength of the leading Borel singularity are determined by the interaction energy of infinitely separated instanton-anti-instanton pairs, and the energy-dependence of the singularity in (18) and (19) comes from the coupling of the external momenta to the quasizeromodes in the classical field. Thus for nontrivial energy-dependence of the large-order behavior to occur it is essential that there exist quasi-zeromodes that couple to the external momenta. The trivial energy-dependence of the large-order behavior in the scalar $\phi^{4}$ theory simply reflects the fact that there are no quasi-zeromodes in the instanton backgrounds that determine the Borel singularity. 


\section{Nonperturbative effects from the perturbative se-} ries

We now show that the Espinosa-Ringwald type cross section can be obtained by taking the minimum element of the perturbative series. This is the reverse process of obtaining the large-order behavior from the Espinosa-Ringwald type cross section. We assume that the coupling $g$ is small. Let us first consider eq. (12). Expanding the integrand about $b=0$ we have,

$$
\tilde{\sigma}(b) \sim \sum_{n=1} \frac{b^{n-1}}{(n-1) !} \int_{a-i \infty}^{a+i \infty} \exp [z(-1+U(x))+(n+\nu-1) \ln z] d z .
$$

For large $n,(26)$ may be evaluated by the saddle point method, giving

$$
c_{n-1} \sim \frac{1}{(n-1) !} \exp [\bar{z}(n)(-1+U(\bar{z}(n)))+(n+\nu-1) \ln \bar{z}(n)],
$$

where $\bar{z}(n)$ is given by

$$
\frac{n+\nu-1}{\bar{z}(n)}+\left.\frac{\partial}{\partial z}(z(-1+U(x)))\right|_{z=\bar{z}(n)}=0 .
$$

Now the perturbative series in (4) is given by

$$
\mathcal{A}\left(p_{i}, g\right)=\sum_{n=1}^{\infty}(n-1) ! c_{n-1}\left(\frac{g}{2 S_{0}}\right)^{n} \sim \sum_{n} e^{F(n, g)}
$$

with

$$
F(n, g)=\dot{z}(n)(-1+U(\bar{z}(n)))+(n+\nu-1) \ln \bar{z}(n)+n \ln \left(\frac{g}{2 S_{0}}\right) .
$$

The minimum element of the perturbative series occurs at $n=\bar{n}$, where $\bar{n}$ satisfies

$$
\left.\frac{\partial}{\partial n} F(n, g)\right|_{n=\bar{n}}=0
$$


Using (28), eq. (31) gives

$$
\bar{z}(\bar{n})=\frac{2 S_{0}}{g} .
$$

Substituting (32) into (30), the minimum element of the perturbative series is given by

$$
\begin{aligned}
a_{\bar{n}} g^{\bar{n}} & \sim \exp [\bar{z}(\bar{n})(-1+U(\bar{x}(\bar{n})))] \\
& =\exp \left[-\frac{2 S_{0}}{g}\left(1-U\left(\frac{\epsilon g}{2 S_{0}}\right)\right)\right],
\end{aligned}
$$

which is the exponential part of the nonperturbative cross section in (8). In deriving (33), we assumed that $\bar{z}(n)$ in (28) is well defined about $n=\bar{n}$ given by (32). It is easy to check that this condition is satisfied if the energy $E$ is small. Thus the perturbative expression for $F$ in $x$ can be obtained by the minimum element of the perturbative series.

If we now slightly modify the argument, we can see that the Espinosa-Ringwald type cross section can also be obtained by doing perturbation of $\tilde{\sigma}(b)$ about the Borel singularity at $b=1$. From the exponential part of (14),

$$
\tilde{\sigma}(b) \sim \exp [\bar{z}(b)(b-1+U(\bar{x}(b)))+\nu \ln \bar{z}(b)]
$$

where $\vec{x}(b)=\epsilon / \bar{z}(b)$ is defined in (13). The coefficients $c_{n-1}$, from the evaluation of (20) with (34) using the saddle point approximation, is given by

$$
c_{n-1} \sim \exp [\bar{z}(\bar{b}(n))(\bar{b}(n)-1+U(\bar{z}(\bar{b}(n))))+\nu \ln \bar{z}(\bar{b}(n))-n \ln \bar{b}(n)]
$$

with $\bar{b}(n)$ being defined by

$$
\bar{z}(\bar{b}(n))-\frac{n}{\bar{b}(n)}=0
$$


In deriving (36), we have used the saddle point equation (13) for $\bar{x}(b)$. Now the perturbative asymptotics for the forward scattering amplitudes is given by

$$
a_{n} g^{n} \sim(n-1) ! c_{n-1}\left(\frac{g}{2 S_{0}}\right)^{n} \sim e^{K}
$$

where

$$
K=\exp \left[\bar{z}(\bar{b}(n))(\bar{b}(n)-1+U(\bar{z}(\bar{b}(n))))+\nu \ln \bar{z}(\bar{b}(n))-n+n \ln \left(\frac{n g}{2 S_{0} \bar{b}(n)}\right)\right]
$$

using Stirling's formula. The minimum element of the perturbative series occurs at order $\bar{n}$, where $\bar{n}$ is defined by

$$
\frac{\bar{n}}{\bar{b}(\bar{n})}=\frac{2 S_{0}}{g}
$$

For (39), we have used (13) and (36). Now from (13) and (39),

$$
\bar{z}(\bar{b}(\bar{n}))=\frac{2 S_{0}}{g}
$$

Substituting (36), (40) into (38), we have

$$
\begin{aligned}
a_{\bar{n}} g^{\bar{n}} & \sim \exp [-\bar{z}(\bar{b}(\bar{n}))(1-U(\bar{x}(\bar{b}(\bar{n})))] \\
& \sim \exp \left[-\frac{2 S_{0}}{g}\left(1-U\left(\frac{\epsilon g}{2 S_{0}}\right)\right)\right]
\end{aligned}
$$

which is again the exponential part of the Espinosa-Ringwald type cross section.

Now in the manipulation through $(34)$ to $(41)$, we have implicitly assumed that $\bar{x}(b)$ given in (13) is well defined, and $\bar{b}(n)$ in (36) is solvable near the saddle point $n=\bar{n}$. It is not difficult to see that these conditions are satisfied if the energy $E$ is small. For example, 
let us assume that the potential $U(x)$ in the standard model is given by the leading term in (11)

$$
U(x)=\frac{1}{2}(3 x)^{\frac{4}{3}}
$$

Then from (13),

$$
1-b=\frac{\nu}{\epsilon} \bar{x}(b)-\frac{1}{6}(3 \bar{x}(b))^{\frac{4}{3}}
$$

It is easy to check that $\bar{x}(b)$ in $(43)$ is solvable in the vicinity,

$$
0 \leq 1-b \leq \frac{9}{32}\left(\frac{\nu}{\epsilon}\right)^{4}
$$

of the Borel singularity at $b=1$. From (40) and (43) we have

$$
1-\bar{b}(\bar{n})=\frac{\nu g}{2 S_{0}}-\frac{1}{6}\left(\frac{3 \epsilon g}{2 S_{0}}\right)^{\frac{4}{3}}
$$

This, combined with (44), gives a bound on the energy

$$
0 \leq \frac{\nu g}{2 S_{0}}\left(1-\frac{\epsilon}{2 \nu}\left(\frac{3 \epsilon g}{2 S_{0}}\right)^{1 / 3}\right) \leq \frac{9}{32}(\nu / \epsilon)^{4}
$$

or

$$
\epsilon \leq \epsilon_{\max }
$$

For $g=4 \pi \alpha_{w}=4 \pi / 30, \epsilon_{\max } \approx 3.96$ The inclusion of higher order terms of $U(x)$ in $(11)$ will not change the qualitative picture, though $\epsilon_{\max }$ may change. Thus it is clear that at low energies we can derive the nonperturbative effects from the perturbative series. In particular, solving $\bar{x}$ in (13) perturbatively in $(1-b)$, we can obtain the exponential part of the Espinosa-Ringwald type cross section given in (8)-(11). Although the Borel transform 
method gives an identical result for the nonperturbative cross section at low energies, we expect a deviation from the usual expression at energies of order $\epsilon_{\max }$.

In the Borel transform method $(1-b)$ is the expansion parameter instead of the energy, and thus there is no direct restriction for the energy to be small, although a new restriction arises from the criterion for the validity of the approximation. Thus the high-energy behavior of the nonperturbative cross section may be better described in the Borel transform method than in the original Espinosa, Ringwald calculation [4]. This point will be illustrated in next section by calculating the anomalous cross section in the standard model using the leading energy-dependent $\tilde{\sigma}(b)$.

\section{The anomalous cross section from the Borel trans-}

\section{form}

Keeping only the leading term of $U(x)$ in (11), and substituting (15) into (14), we have the leading energy-dependent $\tilde{\sigma}(b)$ for the standard model

$$
\tilde{\sigma}(b) \sim \exp \left[\frac{\nu}{2}\left(\frac{3 \epsilon}{\nu}\right)^{\frac{4}{3}}(1-b)^{\frac{1}{3}}-\nu \ln (1-b)\right]
$$

with $\nu$ given in (10). From (35)

$$
c_{n-1} \sim \exp \left[\frac{\nu}{2}\left(\frac{3 \epsilon}{\nu}\right)^{\frac{4}{3}}(1-\bar{b}(n))^{\frac{1}{3}}-\nu \ln (1-\bar{b}(n))-n \ln \vec{b}(n)\right]
$$


with $\vec{b}(n)$ defined by

$$
-\frac{n}{\bar{b}(n)}+\frac{\nu}{1-\bar{b}(n)}-\frac{\nu}{6}\left(\frac{3 \epsilon}{\nu}\right)^{\frac{4}{3}}(1-\bar{b}(n))^{-\frac{2}{3}}=0
$$

The minimum element of the perturbative series is given by

$$
\begin{aligned}
a_{\bar{n}} g^{\bar{n}} & =(\bar{n}-1) ! c_{\bar{n}-1}\left(\frac{g}{2 S_{0}}\right)^{\bar{n}} \\
& \sim \exp \left[\frac{\nu}{2}\left(\frac{3 \epsilon}{\nu}\right)^{\frac{4}{3}}(1-\bar{b}(\bar{n}))^{\frac{1}{3}}-\nu \ln \left(\frac{2 S_{0}(1-\bar{b}(\bar{n}))}{g \nu}\right)-\bar{n}-\nu\right]
\end{aligned}
$$

with

$$
\frac{\bar{n}}{\bar{b}(\bar{n})}=\frac{2 S_{0}}{g}
$$

We added in $(51)\left(\nu \ln \left(g \nu / 2 S_{0}\right)-\nu\right)$ so that the exponential part becomes -1 at $\epsilon=0$. Eq. (51) can be written as,

$$
a_{\bar{n}} g^{\bar{n}} \sim e^{-\frac{4 \pi}{\alpha_{\omega}} F}
$$

where

$$
F=\bar{b}(\bar{n})-\frac{\alpha_{w}}{4 \pi}\left[\frac{\nu}{2}\left(\frac{3 \epsilon}{\nu}\right)^{\frac{4}{3}}(1-\bar{b}(\bar{n}))^{\frac{1}{3}}-\nu \ln \left(\frac{4 \pi(1-\bar{b}(\bar{n}))}{\alpha_{w} \nu}\right)-\nu\right] .
$$

Substituting (52) into $(50), \bar{b}(\bar{n})$ is determined by

$$
\frac{\nu}{1-\bar{b}(\bar{n})}-\frac{\nu}{6}\left(\frac{3 \epsilon}{\nu}\right)^{\frac{4}{3}}(1-\bar{b}(\bar{n}))^{-\frac{2}{3}}=\frac{4 \pi}{\alpha_{w}}
$$

Note the negative sign in the second term of $(55)$. This pushes $\bar{b}(\bar{n})$ toward the Borel singularity, about which $\tilde{\sigma}(b)$ in $(48)$ is valid. Table 1 shows $\bar{x}(\bar{b}(\bar{n}))$ and $F(\bar{b}(\bar{n}))$ for various values of $\epsilon$ from the numerical solution of $\bar{b}(\bar{n})$ in $(55) . F$ decreases very slowly, and is not 
much different from its vacuum value at energies well over the sphaleron energy. In fact, in the limit $\epsilon \rightarrow \infty$, the solution of $(55)$ is

$$
1-\bar{b}(\bar{n})=\frac{8}{3}\left(\frac{\nu}{\epsilon}\right)^{4}
$$

Substituting (56) into (54), we see that $F$ decreases logarithmically at high energies, and thus unitarity is not violated at all reasonably high energies. Note how close $\bar{b}(\bar{n})$ in $(56)$ is to the Borel singularity at $b=1$. This is not the case in the double-well potential or the two-dimensional abelian Higgs model. In these theories, we have

$$
1-\bar{b}(\bar{n})=\frac{\frac{g}{2 S_{0}}(\nu+1+\epsilon / l)}{\bar{b}(\bar{n})+\frac{g}{2 S_{0}}(\nu+1+\epsilon / l)} .
$$

from (21) and (39), which shows that $\bar{b}(\bar{n})$ shifts away from the singularity as $\epsilon$ increases. Thus the approximate Borel transform in (18) of these models is not valid at high energies. Now when does our approximation for (48) of the standard model break down? In this case, it is not as obvious as in the double-well potential or the two-dimensional abelian Higgs model because $\vec{b}(\vec{n})$ stays close to the Borel singularity at all energies. However, in the standard model, the validity of the approximation may be measured by

$$
\delta x=\epsilon^{\frac{4}{3}}(1-b)^{\frac{1}{3}}
$$

in (15), which is an expansion parameter of $\ddot{x}(b)$. For a good approximation, we require

$$
\delta x \ll 1
$$

From Table 1, we see that at $\epsilon \sim 5, \delta x$ becomes of order one. The approximation breaks down at quite low energies, and so beyond these energies the higher-order terms of $U(x)$ 
and $\bar{x}(b)$ in (11), (15) respectively should be included. Thus the unitarity preserving highenergy behavior of $F$ from the leading approximation is not a concrete result. It would be an interesting problem to see how higher-order corrections affect the high-energy behavior of the nonperturbative cross section.

\section{Conclusion}

In summary, we have presented examples of nontrivial energy-dependence of large-order behavior in perturbation theory. A necessary condition for a nontrivial energy-dependence of large-order behavior was shown to be that the classical field that determines the Borel singularity must have quasi-zeromodes coupled to the external momenta. We were also able to show that the Espinosa-Ringwald type cross section can be obtained by taking the minimum element of the perturbative series in the coupling. This suggests that the perturbative series in the coupling is indeed asymptotic. In section 4 we calculated the anomalous cross section in the standard model using the leading energy-dependent Borel transform of the forward scattering amplitudes. It was shown that the validity of the leading approximation for the Borel transform breaks down at relatively low energies. Nevertheless, if we extrapolate the anomalous cross section beyond the energy range where the leading approximation is valid, we find it is exponentially suppresed at all reasonably high energies.

I am grateful to A.H. Mueller and V.I. Zakharov for useful discussions and comments. I am also very thankful to $\mathrm{E}$. Laenen for discussions and reading the manuscript. 


\section{References}

[1] Large-Order Behaviour of Perturbation Theory, ed. J.C. Le Guillou and J. Zinn-Justin. (North-Holland, Amsterdam, 1990).

[2] V.I. Zakharov, Nucl. Phys. B 377 (1992) 501

[3] The Borel singularity of instanton-induced amplitudes, T. Lee, FERMILAB-PUB94/066- T, (to appear in Phys. Lett. B)

[4] O. Espinosa, Nucl. Phys. B 334 (1990) 310; A. Ringwald, Nucl. Phys. B 330 (1990) 1;

[5] W.Y. Crutchfield II, Phys. Rev. D 19 (1979) 2370

[6] M. Mattis, Phys. Rep. 214 (1992) 159.

[7] V.V. Khoze and A. Ringwald, Nucl. Phys. B 356 (1991) 351.

[8] L. McLerran, A. Vainshtein and M. Voloshin, Phys. Rev. D 42 (1990) 171.

[9] S.Yu. Khlebnikov, V.A. Rubakov and P.G. Tinyakov, Nucl.Phys. B 350 (1991) 441 


\begin{tabular}{|c|c|c|c|c|}
\hline$\epsilon$ & $1-\bar{b}(\bar{n})$ & $\bar{x}(\bar{n})$ & $F$ & $\delta x$ \\
\hline 1 & $.1910^{-2}$ & $.2610^{-2}$ & .999 & .125 \\
\hline 5 & $.6810^{-3}$ & $.6910^{-2}$ & .994 & .752 \\
\hline 10 & $.1110^{-3}$ & $.2510^{-2}$ & .989 & 1.033 \\
\hline 100 & $.1310^{-7}$ & $.3110^{-5}$ & .969 & 1.088 \\
\hline 200 & $.8010^{-9}$ & $.3810^{-6}$ & .963 & 1.086 \\
\hline 400 & $.5010^{-10}$ & $.4810^{-7}$ & .956 & 1.085 \\
\hline
\end{tabular}

Table 1: Saddle point values versus energy 